\title{
Polarization axis fluctuations in a single mode VCSEL laser at $780 \mathrm{~nm}$
}

\author{
G. Dudzik, and J. Rzepka \\ Faculty of Electronics, Wroclaw University of Technology, Wyb.Wyspianskiego 27, 50-370 Wroclaw
}

Received August 02, 2013; accepted September 19, 2013; published September 30, 2013

\begin{abstract}
We present a transmission method to determine the polarization axis fluctuations for VCSEL (i.e., Vertical Cavity Surface Emitting Laser). The research is focused mainly on commercial lasers operating at $780 \mathrm{~nm}$, designed for laser spectroscopy. The angle fluctuation $(\alpha)$ of the laser linear axis polarization is elaborated to be less than $150 \mathrm{mrad}$. The measurement errors of this method are studied and calculated. The measurement uncertainty is $\pm 1.4 \mathrm{mrad}$ and this is equivalent to \pm 5 " of arc. Changes of the linear polarization axis depending on the laser current and temperature are presented.
\end{abstract}

The application of VCSEL as a frequency stable coherent radiation source for spectroscopy and interferometry requires linear and time-stable polarization of light. Therefore, it is important to elaborate and implement time-dependent stability measurements. The problems with random polarization of single mode VCSELs were solved by applying azimuth polarization control techniques. These are usually based on semiconductor substrates with (311) crystallographic orientation in the Miller notation [1-3], elliptical shapes of the VCSEL mesa structure [4-5], external mechanical stresses [6] and surface etching technology of diffraction gratings [7]. Up till now, polarization effects in VCSELs are not fully understood and attract a lot of attention in the laser community [8-10]. VCSELs polarization behavior is extremely complicated as a result of many phenomena: polarization switching between linear polarizations, fluctuations, modulations, laser injection current and temperature influences etc. This letter shows an investigation of VCSEL polarization axis fluctuation using a specially built setup to assure similar measurement conditions to those of an interferometer system. Thus, it is possible to verify the polarization behavior in a real measurement system with a fixed laser temperature and a small loopback current signal for frequency stabilization.

In the experimental setup we used a single frequency 780nm VCSEL (ULM780-01-TN-S46FTT, ULM Photonics) dedicated for rubidium spectroscopy with an NTC temperature sensor and a micro Peltier module integrated with the laser structure. The maximum laser current is $3 \mathrm{~mA} @ 0.55 \mathrm{~mW}$ of optical power. A selfdesigned ultra-stable current and temperature laser drivers

$$
\text { *e-mail: grzegorz.dudzik@pwr.wroc.pl }
$$

were used. The driver provides a laser current and temperature stability under $\pm 30 \mathrm{nA}$ and $\pm 0.001^{\circ} \mathrm{C}$, respectively. The linear polarization axis fluctuation has been measured for the laser current set to $2 \mathrm{~mA}$ @ $0.35 \mathrm{~mW}$ and temperature of $20^{\circ} \mathrm{C}$.

The experimental setup for VCSEL polarization stability measurements is depicted in Fig. 1. Laser radiation is divided using an NPBS (i.e., Non Polarized Beam Splitter) creating a measurement and reference channel. The laser beam passes through a polarizer and is collected by a detector (PIN-Si, BPW34). The electrical signal provided by the detector is proportional to the optical power in the measurement channel. When the angle of the VCSEL linear polarization axis is changing, the optical power registered by the detector after the polarizer also changes. Hence, the polarizer is an angle discriminator of the linear polarization in the optical power function.

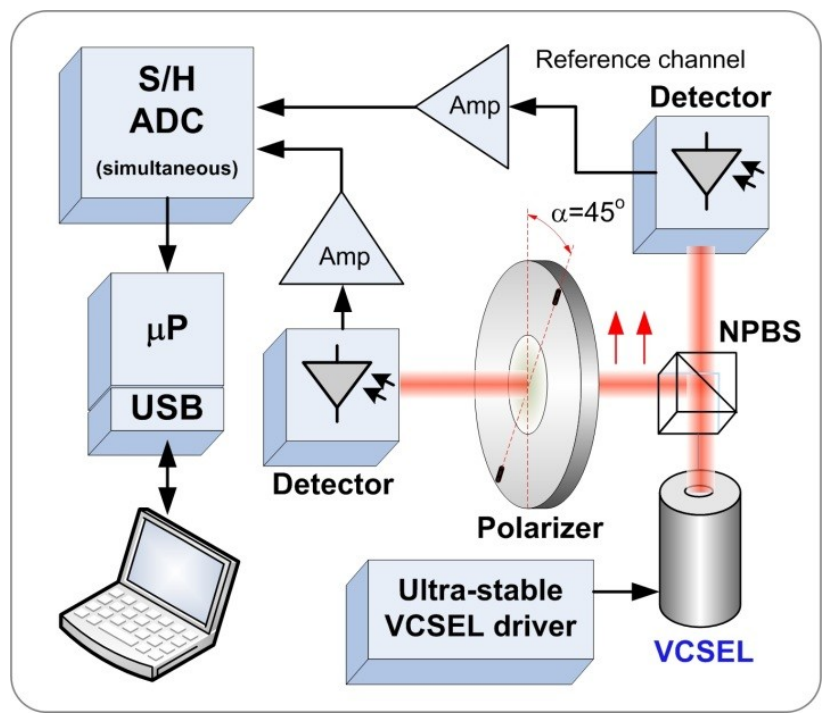

Fig. 1. Experimental setup for measuring VCSEL fluctuation polarization, where: NPBS - Non-Polarized Beam Splitter, Amp amplifier and matching circuit, $\mathrm{S} / \mathrm{H}$ ADC - simultaneous sample-and hold analog-to-digital converter, $\mu \mathrm{P}$ - microprocessor.

To obtain the best linearity and sensitivity of measurements, the main polarizer axis must be shifted by $\alpha=45^{\circ}$ relative to the linear polarization axis of the laser 
beam. For this angle, the polarizer transmittance function has a maximum slope and linearity. The electrical signals are amplified and adapted to 12-bits ADC input level signals. It is essential to administer the same gain coefficient for amplifiers in the measurement and reference channel. Therefore, detected optical power is quantized and represented as a bit number of ADC. We used a sample-and-hold simultaneous ADC converter, microprocessor as a controller and USB interface. The relative power measurement (measuring and reference channel sampled at the same time) allows to minimize the influence of optical power changes, caused by a laser current and temperature fluctuations. The experimental and theoretical characteristics of polarizer transmittance function are determined and presented in Fig. 2. Of course, the polarizer function is proportional to $\cos ^{2}(\alpha)$ according to theoretical Malus law described by the equation:

$$
P_{o p t}=A \cdot \cos ^{2}(\alpha)
$$

where: A - initial intensity, $\alpha$ - angle between light polarization direction and axis of polarizer.

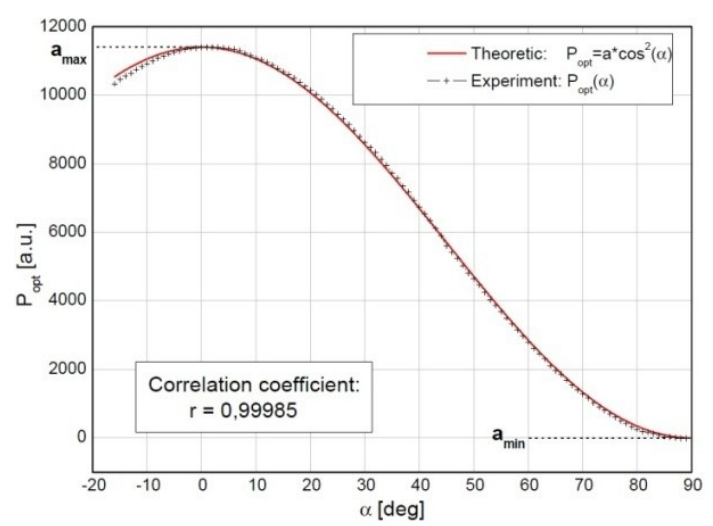

Fig. 2. Theoretical and experimental characteristic of a polarizer transmittance function.

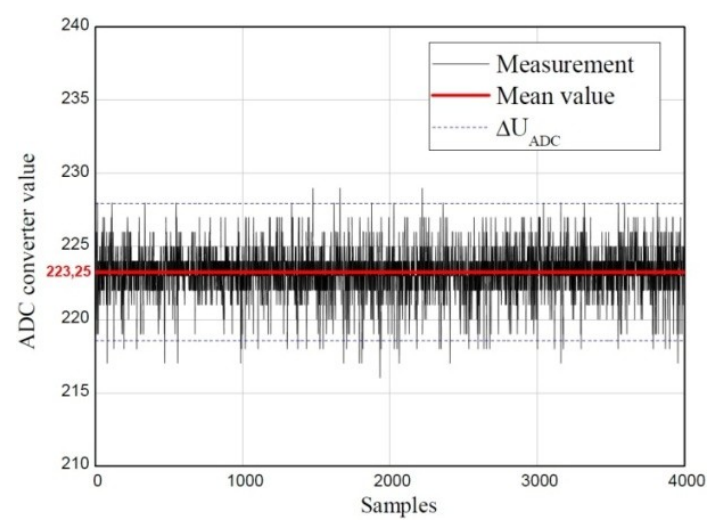

Fig. 3. Electrical noise level in measurement channel.
The value of the measured angle $\alpha$ is burdened with complex measurement uncertainty $\alpha \pm \Delta \mathrm{U}_{\alpha}$, where we should include the assessment of uncertainty input quantities $\Delta \mathrm{P}_{\text {opt }}$ and $\Delta \mathrm{A}$. The calculation of measurement uncertainty of the power $\Delta \mathrm{P}_{\text {opt }}$ should take into account measurement errors due to the noise of detectors with analog circuit $\Delta \mathrm{U}_{\mathrm{S}}$ and $\mathrm{ADC}$ converter nonlinearity $\Delta \mathrm{U}_{\mathrm{ADC}}$ (specified by the manufacturer).

$$
\Delta P_{o p t}=\Delta U_{S}+\Delta U_{A D C}
$$

Therefore, the measurement of signal noise in all of the electrical channels (with no laser radiation) was registered and is depicted in Fig. 3. The standard deviation $S_{p}$ is calculated, based on a series of noise measurements. Because the power measurement uncertainty resulting from the non-linearity of an $\mathrm{ADC}$ converter $\left(\Delta \mathrm{U}_{\mathrm{ADC}}\right)$ is provided by the chip manufacturer in order to improve the same $3 \sigma$ of confidence level, the $\Delta U_{S}$ measurement uncertainty should be taken as: $\Delta U_{S}=3 \cdot S_{p}$ (normal distribution was assumed). The value $\mathrm{A}$ is the difference between $\mathrm{a}_{\max }$ and $\mathrm{a}_{\min }$ on characteristic $\mathrm{P}_{\text {opt }}(\alpha)$ shown in Fig. 2. Because each of the value $a_{\min }$ and $a_{\max }$ is burdened with errors resulting from $\mathrm{ADC}$ converter nonlinearity $\Delta \mathrm{U}_{\mathrm{ADC}}$, the uncertainty of the input quantity $\Delta \mathrm{A}$ is given by the equation:

$$
\Delta A=\Delta a_{\max }+\Delta a_{\min }=2 \cdot \Delta U_{A D C}
$$

An exact differential method was used to calculate angle $(\alpha)$ measurement uncertainty described by the following:

$$
\begin{array}{r}
\Delta U_{\alpha}=\left|\frac{-1}{\sqrt{1-\frac{P_{o p t}}{A}}} \cdot \frac{1}{2 \sqrt{\frac{P_{o p t}}{A}}} \cdot \frac{1}{A}\right| \cdot \Delta P_{o p t} \\
+\left|\frac{-1}{\sqrt{1-\frac{P_{o p t}}{A}}} \cdot \frac{1}{2 \sqrt{\frac{P_{o p t}}{A}}} \cdot \frac{-P_{o p t}}{A^{2}}\right| \cdot \Delta A
\end{array}
$$

The values of parameters required to calculate $\Delta U_{\alpha}$ are presented in Table 1 . The angle measurement uncertainty is calculated using Eq. (4) and it is equal to $\Delta U_{\alpha}=$ $1.4058[\mathrm{mrad}] \cong \mathbf{0}^{\circ} \mathbf{0}^{\prime} \mathbf{5}^{\prime \prime}$.

Table 1. The parameter values for the calculation of polarization angle measurement uncertainty.

\begin{tabular}{|c|c|c|c|}
\hline Parameter & Value & Parameter & Value \\
\hline $\mathrm{S}_{\mathrm{p}}$ & 1.57 & $\Delta \mathrm{U}_{\mathrm{ADC}}$ & 1.5 \\
\hline$\Delta \mathrm{U}_{\mathrm{S}}$ & 4.7 & $\Delta \mathrm{A}$ & 3 \\
\hline$\Delta \mathrm{P}_{\mathrm{opt}}$ & 6.2 & $\mathrm{~A}$ & 11403 \\
\hline $\mathrm{P}_{\mathrm{opt}}$ & 5893 & \multicolumn{3}{|l}{} \\
\hline
\end{tabular}

The optical power $\mathrm{P}_{\text {opt }}$ measurement (angle $\alpha$ indirectly) was made automatically with full data acquisition and was not affected by external ambient light. The results of 
measuring power in both channels are depicted in Fig. 4. whereas angle fluctuations of the laser polarization $\alpha$ are presented in Fig. 5. The maximum changes of VCSEL polarization angle $\Delta \alpha$ registered during one hour are given as the difference between $\alpha_{\max }$ and $\alpha_{\min }$ described as $\Delta \alpha_{(1 \mathrm{~h})}=\alpha_{\max }-\alpha_{\min }=147.71$ [mrad] $=8 ' 51$ '. Because both values $\alpha_{\max }$ and $\alpha_{\min }$ are burdened by the same error $\Delta \mathrm{U}_{\alpha}$, the VCSEL laser linear axis polarization fluctuations can be expressed as:

$$
\Delta \alpha_{(1 h)} \pm 2 \cdot \Delta U_{\alpha}=8^{\prime} 51 " \pm 0^{\prime} 10^{\prime \prime}
$$

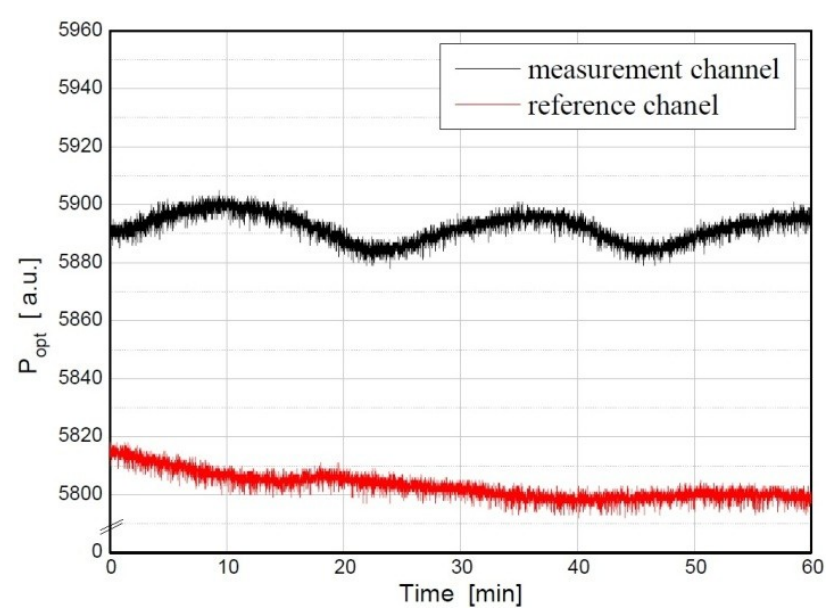

Fig. 4. Registered optical power fluctuations for reference and measurement channel in the time domain.

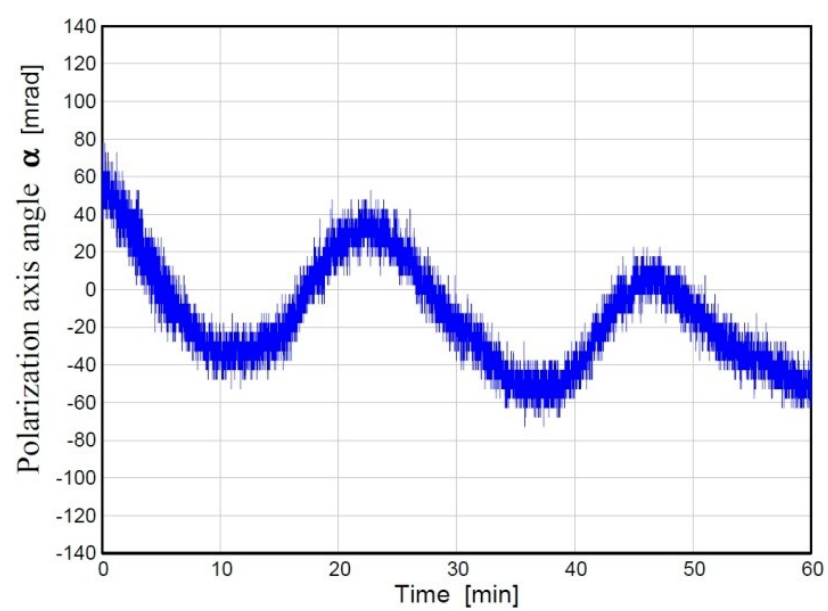

Fig. 5. Registered VCSEL polarization axis fluctuations.

The obtained results of polarization axis fluctuation under $150 \mathrm{mrad}$ are sufficient enough for interferometry and spectroscopy applications. But very interesting are visible long term fluctuations (Fig. 5) with a period of around $25 \mathrm{~min}$. This is not exactly a polarization switching effect because according to the papers [10-11], it is very quick (few $\mu \mathrm{s}$ ) and has a rather step character. It does not mean it is not present here. We think that the reason could be the influence of an injection laser current [12] and temperature changes. A similar effect is observed only when the laser current is swept and the polarizer is placed in an optical path. When the current is slowly increased the long term fluctuations appear, which is depicted in Fig. 6. Additionally, a strong polarization effect is observed when the laser temperature is changed. For temperature accretion $\Delta \mathrm{T}=8^{\circ} \mathrm{C}$, linear polarization axis direction is rotated over $2 \mathrm{deg}$. We suppose that the presented two strong effects - depending on temperature and injection current of the laser - are mainly responsible for periodic long term polarization instability.

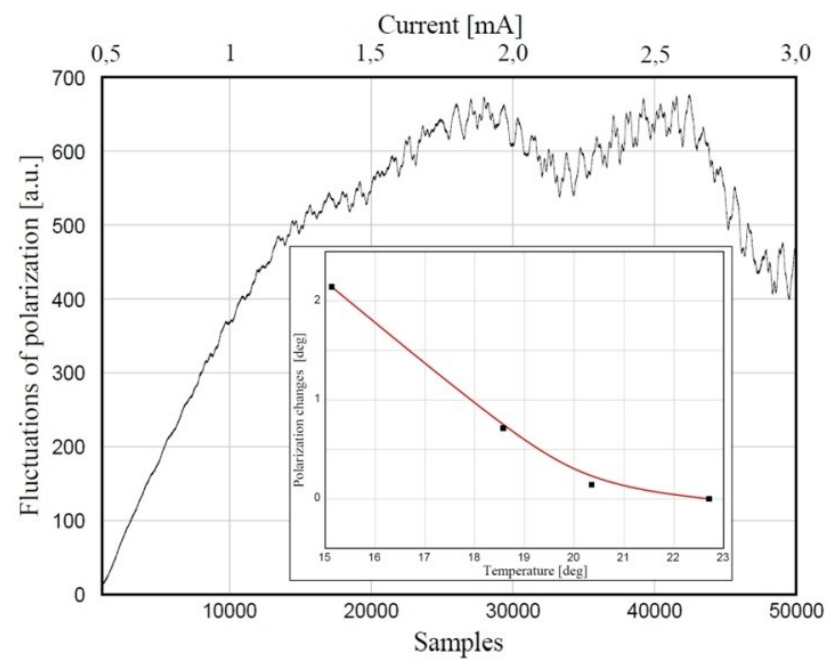

Fig. 6. Polarization fluctuations during a current sweep and laser temperature changes.

In conclusion, we have presented an experimental setup for studying the polarization axis fluctuations of VCSELs. We estimated the angular instability of linear axis polarization to be about $150 \mathrm{mrad}$. Also, the presented measurement method accuracy is defined and equal to $\pm 1.4 \mathrm{mrad}$.

We acknowledge the support from the Lasertex Ltd. and the Department of Electronics, Wroclaw University of Technology.

\section{References}

[1] T. Ohtoshi et al., Appl. Phys. Lett. 63, 1886 (1994).

[2] K. Tateno et al., Appl. Phys. Lett. 70, 3395 (1997).

[3] O. Tadanaga et al., IEEE Photon. Tech. Lett. 12, 942 (2000).

[4] K. Choquette, R. Leibenguth, IEEE Photon. Tech. Lett. 6, 40 (1994).

[5] B. Weigl et al., IEEE J. Sel. Top. Quantum Electron. 3, 409 (1997).

[6] P. Dowd et al., Electron. Lett. 33, 1315 (1997).

[7] M. Grabherr et al., Proc. SPIE, 6908, 690803 (2008).

[8] R. Roy et al., Phys. Rev. Lett. 45, 1486 (1980).

[9] J. Martín-Regalado et al., Appl. Phys. Lett. 70, 3350 (1997).

[10] M. B. Willemsen et al., Phys. Rev. Lett. 82, 4815 (1999).

[11] M. B. Willemsen et al., Phys. Rev. Lett. 84, 4337 (2000).

[12] T. Mukaihara et al., IEEE Photon. Technol. Lett. 7, 1113 (1995). 